\title{
Effect of Different Surface Treatments on Repair Bond Strength of Aged Tooth Colored Materials
}

\author{
Faika Y. Abdelmegid ${ }^{1}$ \\ ${ }^{I}$ Department of Oral Medicine and Diagnostic Sciences, King Saud University, College of Dentistry
}

\begin{abstract}
:
Objectives: The aim of this investigation was to assess the influence of different surface treatments and repair material on the repair shear bond strength (SBS) of GC Fuji IX GP Packable Posterior Restorative, Filtek Bulk Fill, and Filtek Supreme Ultra Flowable.

Methods: Thirty cylindrical specimens were prepared from each material and randomly allocated into 3 groups/10 each. Group 1: no surface treatment (control), group 2 (treatment 1) where a GC G-Bond was applied while group 3 (treatment 2) the G-Bond was applied after roughening the surface with a finishing diamond bur. All specimens were bonded to Heliomolar Flow. The SBS was measured using a universal testing machine.

Results: There were significant effects of surface treatments, materials, and interaction ( $p<0.01)$. For GC Fuji $I X G P$ and Filtek Bulk Fill; treatment 2 exhibited significantly higher SBS than either no surface treatment or treatment 1 which were not statistically significantly different from each other. For Filtek Supreme Ultra Flowable; there was a statistically significant difference in SBS between all treatments.

Conclusion: Application of self-etching adhesive after roughening the surface with a finishing diamond bur produces the highest repair SBS for GC Fuji IX GP, Filtek Bulk Fill, and Filtek Supreme Ultra Flowable.
\end{abstract}

Keywords: Shear bond strength, surface conditioning, aging, repair, resin composite

\section{Introduction}

Replacement of dental restorations is greater in children, and highest in the primary teeth. ${ }^{[1]} \mathrm{A}$ survey reported that repair of restorations is taught in some dental schools in the United States, Canada and Puerto Rico and that patients were willing to accept such treatment. ${ }^{[2]}$ Most schools considered the repair of resin composite restorations to be a definitive measure and reported that, on average, they expected a repaired resin-based composite restoration to have a longevity of four years. ${ }^{[2]}$ Durable repair of failed restorations is critical for future clinical performance, survival and high success rate ${ }^{[1] \&[2]}$ The investigation of proper techniques for repair is critically important. ${ }^{[1]}$ Methods of enhancement of bond strength between the old restorations and repair materials such as increasing the surface roughness with green silicon carbide burs and coating of old restorations with bonding agents have been reported. ${ }^{[3],[4] \&[5]}$ However, the use of the new possible repair materials and different surface treatments on repair restorations has not been sufficiently studied.

Approximately 90 million new restorations are put in in the United States yearly and 200 million are replaced ${ }^{[6]}$ Dental restorations with shortcomings could be managed by replacement, repair or no repair. ${ }^{[7]}$ Repair will cause less iatrogenic damage than complete replacement, and is therefore preferred. A major goal of repair is the enhancement of adhesion between the new and old restorative materials and tooth structure. High resin-based composite repair bond strength is essential ${ }^{[8]}$ Replacement of defective restorations with new ones cause dentists to devote nearly half of their chair time replacing restorations, which involves the risk of removing sound tooth substance. ${ }^{[9] \&[10]}$ Therefore, conservative techniques for repairing defective restorations have been proposed because it is easy, quick, simple, and cost effective..$^{[11],[12],[13],[14] \&[15]}$ Small defects of stained and degraded margins may be removed by refurbishing/refinishing procedures. ${ }^{[14]}$

It is important to find out if there is any effect of surface conditioning methods of restorations and repair materials to improve bond strength in vitro. What is the best method of surface treatment and repair material that may produce high repair bond strength between the restorative material and repair material is a question that has not been satisfactorily answered. The significance of this study is that although the repair of existing restorations is advocated, there is a paucity of research data to support the use of specific products and techniques to improve the repair bond strength. Regardless of the extensive research on the effectiveness of repaired restorations, little research has addressed the influence of the methods of surface treatment of dental restorations and materials used for repair. Therefore, the aim of this investigation was to assess the influence of different surface treatments and a repair material on the repair shear bond strength of a packable posterior glass ionomer (GC Fuji IX GP Packable Posterior Restorative), a bulk fill posterior resin composite (Filtek Bulk Fill), 
and a flowable resin composite (Filtek Supreme Ultra Flowable). The null hypothesis was that no difference in repair bond strength of different restorative materials using different methods of surface treatment.

\subsection{Materials and preparation of specimens}

\section{Materials And Methods}

One packable posterior glass ionomer (GC Fuji IX GP Packable Posterior Restorative), a bulk fill posterior resin composite (Filtek Bulk Fill), and a flowable resin composite (Filtek Supreme Ultra Flowable) were used in this study (Table 1).Thirty disk specimens ( $8 \mathrm{~mm}$ diameter, $2 \mathrm{~mm}$ thickness) were prepared from each material according to the instructions of the manufacturers. Each material was dispensed and condensed in the Teflon mold according to the instructions of the manufacturers. Immediately the mix was covered with a Mylar strip and a glass slide and stabilized using a C-clamp. Where applicable, the materials were polymerized using a curing light (Bluephase, Ivoclar Vivadent) with a light intensity of 1,200 $\mathrm{mW} / \mathrm{cm} 2$ placed as closely as possible to the surface of each material. Then the Mylar strip was removed and no further trimming or polishing was carried out.The surface of each specimen was wet-ground flat with 600-grit silicon carbide paper and then were stored in closed containers of $20 \mathrm{ml}$ distilled water at $37^{\circ} \mathrm{C}$ for 6 months. All specimens were thermocycled for 1000 rounds between $5^{\circ} \mathrm{C}$ and $55^{\circ} \mathrm{C}$ temperatures (dwell time $=20 \mathrm{~s}$, transfer time $=10 \mathrm{~s}$ ) based on the standards of the International Organization for Standardization). ${ }^{[16]}$ The specimens from each material were randomly allocated into 3 groups/10 each (Table 2) and stored in distilled water at $25^{\circ} \mathrm{C}$ for $24 \mathrm{~h}$.

\subsection{Surface treatment procedures}

The surface treatment of materials tested is listed in Table 2. Ten specimens of each material were used as control with no surface treatment. Group 2 (treatment 1) where GC G-Bond was applied while group 3 (treatment 2) GC G-Bond was applied after roughening the surface with a finishing diamond bur (Coarse, Cat. No. C13 Strauss Diamond Instruments Inc. Westport, CT) for 10 seconds. All specimens were placed in the assembly device and a flowable microfilled resin composite (Heliomolar Flow) was inserted into the split Teflon mold and cured by exposure to visible light for 40 seconds and stored for 48 hours in closed containers of $20 \mathrm{ml}$ distilled water at $37^{\circ} \mathrm{C}$.

\subsection{Measurements of bond strength}

The SBS was measured for each specimen in a universal testing machine (model no. 8500, Instron, Canton, MA, USA) at crosshead speed of $0.5 \mathrm{~mm} / \mathrm{min}$. Bond strength was expressed in MPa.

\subsection{Statistical analysis}

Descriptive statistics of the SBS and analysis of data was completed using one-way ANOVA to compare the mean values of these outcome variables, followed by Tukey's post hoc test for multiple comparisons of mean values. All statistical analyses were set with a significance level of $p<0.05$. The statistical analysis was performed with SPSS Version 16.0 (SPSS Inc. Released 2007. SPSS for Windows, Chicago, SPSS Inc., Ill).

\section{Results}

Table 3 shows descriptive statistics of the SBS for each material by treatment group. Within each material no treatment (control) produced the lowest SBS and Filtek Bulk Fill produced the highest SBS, but the magnitude of the difference in strength between different treatments differed with material. Within treatment, Fuji IX GP produces the lowest SBS and Filtek Supreme Ultra Flowable produces the highest SBS.

There were significant effects of surface treatments, materials, and interaction $(p<0.01)$. For GC Fuji IX GP and Filtek Bulk Fill; treatment 2 exhibited significantly higher SBS than either no surface treatment or treatment 1 which were not statistically significantly different from each other. For Filtek Supreme Ultra Flowable; there was a statistically significant difference in SBS between all treatments.

For Fuji IX GP and Filtek Bulk Fill, no statistically significant difference in SBS between no treatment and treatment1. Treatment 2 showed significantly greater SBS than either no treatment or treatment 1 . For Filtek Supreme Ultra Flowable, there was a statistically significant difference in SBS between all treatments with no treatment having lower SBS than treatment 1 or treatment 2 and treatment 1 having lower SBS than treatment 2 .

Within the no treatment group, there was a statistically significant difference in SBS between Fuji IX GP and Filtek Bulk Fill with Fuji IX GP having lower SBS. There was also a significant difference between Fuji IX GP and Filtek Supreme Ultra Flowable, with Fuji IX GP having lower SBS. No significant difference between Filtek Bulk Fill and Filtek Supreme Ultra Flowable. Within treatment 1, there was a statistically significant difference in SBS between all materials with Fuji IX GP having lower SBS than Filtek Bulk Fill or Filtek Supreme Ultra Flowable and Filtek Bulk Fill having lower SBS than Filtek Supreme Ultra Flowable. 
Within treatment 2, there was a statistically significant difference in SBS between all materials with Fuji IX GP having lower SBS than Filtek Bulk Fill or Filtek Supreme Ultra Flowable and Filtek Bulk Fill having lower SBS than Filtek Supreme Ultra Flowable.

\section{Discussion}

The null hypothesis was rejected as there was a difference in repair bond strength of different restorative materials using different methods of surface treatment. Numerous elements play a part in the choice of repair as a substitute to replacement of failed and unsuccessful restorations such as the involved restorative material, assessments of cost and benefit, the clinical circumstance, the feature of the present restoration, the repair strength needed, the biological value of a total replacement, and the abilities of the operator. ${ }^{[15]}$

Composites undergo degradation over time. ${ }^{[17]}$ Investigators have compared repair bond strength of resin modified glass ionomer cement, poly-acid-modified composite resin, and resin composite using different adhesion primers and different surface treatment methods and concluded that some adhesion primers and some surface treatment methods yielded higher bond strength than others. ${ }^{[3],[4],[5],[8],[18] \&[19]}$ The time of repair significantly affected the bond strength. ${ }^{[20] \&[21]}$ Two recent studies have compared the repair potential of resinbased composite using self-etching adhesive and reported contradictory results in supporting the self-etching adhesive. ${ }^{[22] \&[23]}$ A study examined the influence of preparation and bonding methods on the reliability of repaired resin composite reported that flowable resin composite showed great quality of the margin without being statistically significant. ${ }^{[9]}$ It has been reported that repair bond strength may attain up to $65 \%$ of the cohesive strength of intact resin composite ${ }^{[18] \&[20]}$ Roughening resin composite with sandblasting and green silicon carbide burs are promising approaches to accomplish worthy resin-resin bonds. ${ }^{[3] \&[4]}$ Nevertheless, the use of new materials and surface treatments on repair restorations have not been studied.

Although several studies have assessed the influence of surface treatments and different parameters of old and freshly placed restorations. ${ }^{[24] \&[25]}$ little data is available investigating the bond strength associated with the repair of the new restorative materials and the new bonding agents. The present study showed that there is a difference between various methods of surface treatments of tested restorative materials and the repair shear bond strength. In addition, the use of bonding agent alone enhanced the repair bond strength while the use of a diamond bur and bonding agent enhanced the repair bond strength significantly. Also, there was a difference in repair bond strength between the materials tested. It was reported that microhybrid resin composite have greater repair strengths than nanofilled resin composites and sandblasting produced the highest bond strength. ${ }^{[26]}$

Repair using of diamond burs for preparing the surface of resin composite for bonding have shown divergent results. ${ }^{[26] \&[27]} \mathrm{A}$ study showed higher bond strength of the repair when diamond bur was used compared to sandblasting. ${ }^{[26]}$ In the present investigation we used a coarse diamond bur (average diamond grit of $100 \mathrm{um}$ ) that was expected to produce very irregular surface roughness. Possibly an additional retentive surface may be accomplished using a finer grit bur. In the present study, the Mylar strip was used with no further trimming or polishing. Mylar strips generate reproducible and smooth surfaces. ${ }^{[28]}$ The repair bond strengths of polymerized and unpolymerized resin composites against Mylar strips were not significantly different. ${ }^{[28]}$ However, another study reported slightly greater cohesive failure when resin composite was not polymerized against Mylar strips than with the Mylar strip. ${ }^{[28]}$ The influence of the presence of the oxygen inhibited layer on the repair of resin composite is controversial ${ }^{[29]}$ and nullify by the absence of a significant difference in bond strength between polymerization in the presence and absence of a Mylar strip. ${ }^{[30]}$ This indicates that an oxygen inhibited layer is not necessary for bonding to composites. ${ }^{[28]}$

The use of an intermediate bonging resin showed non-significant increases in repair bond strength when intermediate bonging resin was used. ${ }^{[28]}$ In contrast, our study showed that intermediate bonging resin increased repair bond strength. Earlier studies showed that the repair bond strength of aged resin composites increased after intermediate bonding resin application. ${ }^{[31] \&[32]}$ However, another study showed no increase when intermediate bonding resin is applied on fresh resin composite. ${ }^{[2]}$ This is probably due to the degree of conversion in fresh resin composites is high and positive properties of the intermediate bonding resin do not become obvious. ${ }^{[28]}$ Clinically it is important to expose the restorative materials to the oral environment before repairing to validate the aging influences on the chemical and physical properties of the resin composites and subsequently on adhesion. In the present study, the specimens were aged by storing in closed containers of 20 $\mathrm{ml}$ distilled water at $37^{\circ} \mathrm{C}$ for 6 months and then thermocycled for 1000 rounds. A study reported that repair bond strength of nanofilled resin composites decreased after thermocycling but no significant difference was noted between the two resin composites tested. ${ }^{[33]}$ This reduction may be due to the bis-GMA and TEGDMA in the nanohybrid resin composite which affect water absorption. ${ }^{[34]}$ 
In this study, no attempt was done to assess the debonded specimens to determine the mode of failure. Another study showed that when the adhesive strength at the joint interface surpassed the cohesive strength of the resin composite it indicates the clinical reliability of the adhesion ${ }^{[28]}$ and when a resin composite repaired inclines to fracture cohesively, a better durable adhesion can be expected under occlusal load. ${ }^{[34]}$ Also, materials with similar bond strengths do not show the same types of failure. ${ }^{[28]}$

\section{Conclusions}

Under the experimental conditions and within the limitations of this investigation, these conclusions can be drawn:

1. Treatment of the surface of tested material with a diamond bur and applying self-etching adhesive resulted in the highest repair bond strength.

2. Within each material no treatment (control) produced the lowest SBS.

3. Within treatment, Fuji IX GP produces the lowest SBS and Filtek Supreme Ultra Flowable produces the highest SBS.

\section{Acknowledgment}

The authors would like to thank the College of Dentistry Research Center and Deanship of Scientific Research at King Saud University, Saudi Arabia for funding this research project.

\section{References}

[1]. L. Wendt, G. Koch, and D. Birkhed. Replacements of restorations in the primary and young permanent dentition. Swed Dent J 22(4), 1998, 149-155.

[2]. V. Gordan,I. Mjör, I. Blum, and N. Wilson. Teaching Students the Repair of Resin-Based Composite Restorations: A Survey of North American Dental Schools. J Am Dent Assoc 134(3), 2003, 317-323.

[3]. E. Swift EJ, B. LeValley, and D. Boyer. Evaluation of new methods for composite repair. Dent Mater 8(6), 1992, 362-365.

[4]. E. Swift Jr, B. Cloe, D. Boyer. Effect of a silane coupling agent on composite repair strengths. Am J Dent 7(4), 1994, 200-202.

[5]. R. Frankenberger, N. Kramer, J. Ebert, U. Lohbauer, S. Kappel, S. ten Weges, and A. Petschelt. Fatigue behavior of the resin-resin bond of partially replaced resin-based composite restorations. Am J Dent 16(1), 2003, 17-22.

[6]. P. Murray, I. About, J. Franquin, M. Remusat, and A. Smith. Restorative pulpal and repair responses. J Am Dent Assoc 132(4), 2001, 482-491.

[7]. C. Penning. Repair and revision 1. Repair or replacement of amalgam. Ned Tijdschr Tandheelkd 108(2), 2001, 46-49.

[8]. A. Tezvergil, L. Lassila, and P. Vallittu. Composite-composite repair bond strength: effect of different adhesion primers. J Dent 31(8), 2003, 521-525.

[9]. R. Frankenberger, S. Roth, N. Kramer, M. Pelka, and A. Petschelt. Effect of preparation mode on Class II resin composite repair. J Oral Rehabil 30(6), 2003, 559-564.

[10]. R. Going, and M. Jendreson. Failure related to materials used in restorative dentistry. Dent Clin North Am 16(1), 1972, 71-86.

[11]. T. Kallio, T. Lastumaki, and P. Vallittu. Bonding of restorative and veneering composite resin to some polymeric composites. Dent Mater 17(1), 2001, 80-86.

[12]. L. Baratieri,S. Monteiro Jr, and M. Andrada. Amalgam repair: a case report. Quintessence Inter 23(8), 1992, 527531.

[13]. J. Summitt, J. Osborne, J. Burgess, and M. Howell. Effect of grooves on resistance form of Class 2 amalgams with wide occlusal preparations. Oper Dent 18(2), 1993, 42-47.

[14]. I. Mjor, and V. Gordan. Failure, repair, refurbishing and longevity of restorations. Oper Dent 27(5), 2002, 528-534.

[15]. I. Mjor. Repair versus replacement of failed restorations. Int Dent J 43(5), 1993, 466-472.

[16]. International Organization for Standardization. ISO TR 11405. Dental material guidance on testing of adhesion to tooth structure. 1994.

[17]. J. Ferracane. Hygroscopic and hydrolytic effects in dental polymer networks. Dent Mater 22(3), 2006, 211-222.

[18]. C. Sau,G. Oh, H. Koh, C. Chee, and C. Lim. Shear bond strength of repaired composite resins using a hybrid composite resin. Oper Dent 24(3), 1999, 156-161.

[19]. M. Ozcan, P. Alander, P. Vallittu, M. Huysmans, and W. Kalk. Effect of three surface conditioning methods to improve bond strength of particulate filler resin composites. J Mater Sci Mater Med 16(1), 2005, 21-27.

[20]. G.Lewis, W. Johnson, W. Martin, A. Canerdy, C. Claburn, and M. Collier. Shear bond strength of immediately repaired light-cured composite resin restorations. Oper Dent 23(3), 1998, 121-127.

[21]. R. Shaffer, D. Charlton, and C. Hermesch. Repairability of three resin-modified glass-ionomer restorative materials. Oper Dent 23(4), 1998, 168-172.

[22]. W.Dias, A. Ritter, and E. Swift Jr. Repairability of a packable resin-based composite using different adhesives. Am J Dent 16(3), 2003, 181-185. 
[23]. X. Niu, and X. Wang. Comparison of the clinical efficacy of self-etching bond system and composite resin in repair of dental defects. Shanghai Kou Qiang Yi Xue 13(6), 2004, 487- 489.

[24]. S.Myaki, C. Rodrigues, D. Raggio, T. Flores, and M. Matson. Microleakage in primary teeth restored by conventional or bonded amalgam technique. Braz Dent J 12(3), 2001, 197-200.

[25]. A. Yap, K. Lye, and C. Sau. Effects of aging on repair of resin-modified glass-ionomer cements. J Oral Rehabil 27(5), 2000, 422-427.

[26]. N. Nassoohi, H. Kazemi, M. Sadaghiani, M. Mansouri M, and V. Rakhshan. Effects of three surface conditioning techniques on repair bond strength of nanohybrid and nanofilled composites. Dent Res J (Isfahan) 12(6), 2015, 554561.

[27]. F. Papacchini, S. Dall'Oca, N. Chieffi, C. Goracci, F. Sadek, B. Suh, F. Tay, and M. Ferrari. Composite-to-composite microtensile bond strength in the repair of a microfilled hybrid resin: effect of surface treatment and oxygen inhibition. J Adhes Dent 9(1), 2007, 25-31.

[28]. M. Rinastiti, M. Ozcan, Siswomihardjo W, and H. Busscher. Immediate repair bond strengths of microhybrid, nanohybrid and nanofilled composites after different surface treatments. J Dent 38(1), 2010, 29-38.

[29]. S. Dall'Oca, F. Papacchini, C. Goracci, A. Cury, B. Suh, F. Tay, A. Polimeni, and M. Ferrari. Effect of oxygen inhibition on composite repair strength over time. J Biomed Mater Res B Appl Biomater81(2), 2007, 493-498.

[30]. B. Suh. Oxygen-inhibited layer in adhesion dentistry. J Esthet Restor Dent16(5), 2004, 316-323.

[31]. S. Shahdad, and J. Kennedy. Bond strength of repaired composite resins: an in vitro study. J Dent 26(8), 1998, 685694.

[32]. A. Tezvegril, L. Lassila, and P. Vallittu. Composite-composite repair bond strength: effect of different adhesion primers. J Dent31(8), 2003, 521-525.

[33]. M. Özcan, P. Corazza, S. Marocho, S. Barbosa, and M. Bottino. Repair bond strength of microhybrid, nanohybrid and nanofilled resin composites: effect of substrate resin type, surface conditioning and ageing. Clin Oral Investig;17(7), 2013, 1751-1758.

[34]. I. Sideridou, V. Tserki, G. Papanastasiou. Study of water sorption, solubility and modulus of elasticity of light-cured dimethacrylate-based dental resins. Biomaterials 24(4), 2003, 655-665.

[35]. C. Lucena-Martin, S. Lopez, and J. de Mondelo. The effect of various surface treatments and bonding agents on the repaired strength of heat-treated composites. J Prosthet Dent 86(5), 2001, 481-488.

Table 1. Materials used in this Study

\begin{tabular}{|l|l|l|}
\hline Product/Material & USE & Manufacturer \\
\hline $\begin{array}{l}\text { GC Fuji IX GP Packable Posterior } \\
\text { Restorative }\end{array}$ & Repair substrate & GC America Inc., Alsip, IL, USA \\
\hline Filtek Bulk Fill Posterior Restorative & Repair substrate & 3M ESPE Division, St. Paul, MN, USA \\
\hline $\begin{array}{l}\text { Filtek Supreme Ultra Flowable } \\
\text { Restorative }\end{array}$ & Repair substrate & 3M ESPE Division, St. Paul, MN, USA \\
\hline GC G-Bond & Intermediate resin & GC America Inc., Alsip, IL, USA \\
\hline Heliomolar Flow & Repair resin & Ivoclar Vivadent Inc., Amherst, NY, USA \\
\hline
\end{tabular}

Table 2. Distribution of materials and surface treatments used in this study

\begin{tabular}{|l|l|l|}
\hline $\begin{array}{l}\text { Group/Subgroup } \\
\text { Number }\end{array}$ & Repair Substrate Material & Surface Treatment + Repair Resin \\
\hline $1 \mathrm{a}$ & Fuji IX GP & No Treatment (Control) + Heliomolar Flow \\
\hline $1 \mathrm{~b}$ & Fuji IX GP & GC G-Bond + Heliomolar Flow \\
\hline $1 \mathrm{c}$ & Fuji IX GP & Diamond bur + GC G-Bond + Heliomolar Flow \\
\hline $2 \mathrm{a}$ & FILTEK BULK FILL & No Treatment (Control) + Heliomolar Flow \\
\hline $2 \mathrm{~b}$ & FILTEK BULK FILL & GC G-Bond + Heliomolar Flow \\
\hline $2 \mathrm{c}$ & FILTEK BULK FILL & Diamond bur + GC G-Bond + Heliomolar Flow \\
\hline $3 \mathrm{a}$ & Filtek Supreme Ultra Flowable & No Treatment (Control) + Heliomolar Flow \\
\hline $3 \mathrm{~b}$ & Filtek Supreme Ultra Flowable & GC G-Bond + Heliomolar Flow \\
\hline $3 \mathrm{c}$ & Filtek Supreme Ultra Flowable & Diamond bur + GC G-Bond + Heliomolar Flow \\
\hline
\end{tabular}


Table 3. Shear bond strength for each material by treatment group*

\begin{tabular}{||l|l|c|c|c|c||}
\hline $\begin{array}{l}\text { Repair Substrate } \\
\text { Material }\end{array}$ & Surface Treatment + Repair Resin & Mean & Std & Min & Max \\
\hline Fuji IX GP & No Treatment (Control) + Heliomolar Flow & 5.74 & 0.63 & 4.34 & 6.15 \\
\hline Fuji IX GP & GC G-Bond + Heliomolar Flow & 6.61 & 0.81 & 5.32 & 7.56 \\
\hline Fuji IX GP & Diamond bur + GC G-Bond + Heliomolar Flow & 8.76 & 0.98 & 7.53 & 10.20 \\
\hline Filtek Bulk Fill & No Treatment (Control) + Heliomolar Flow & 8.46 & 1.15 & 6.45 & 10.01 \\
\hline Filtek Bulk Fill & GC G-Bond + Heliomolar Flow & 9.77 & 1.19 & 8.40 & 11.58 \\
\hline Filtek Bulk Fill & Diamond bur + GC G-Bond + Heliomolar Flow & 15.10 & 1.43 & 13.07 & 16.90 \\
\hline $\begin{array}{l}\text { Filtek Supreme Ultra } \\
\text { Flowable }\end{array}$ & No Treatment (Control) + Heliomolar Flow & 9.37 & 0.83 & 8.10 & 10.38 \\
\hline $\begin{array}{l}\text { Filtek Supreme Ultra } \\
\text { Flowable }\end{array}$ & GC G-Bond + Heliomolar Flow & 12.96 & 1.38 & 10.71 & 14.55 \\
\hline $\begin{array}{l}\text { Filtek Supreme Ultra } \\
\text { Flowable }\end{array}$ & Diamond bur + GC G-Bond + Heliomolar Flow & 17.60 & 1.99 & 14.72 & 19.80 \\
\hline
\end{tabular}

$* \mathrm{~N}=10$ for each group 